\title{
NEUROIMAGING IN NEWLY DIAGNOSED EPILEPSY
}

The use of neuroimaging and its value in diagnosis were determined in a community based study of 613 children with newly diagnosed epilepsy referred to Yale University, New Haven, CT. Of the patients examined, $80 \%$ had imaging studies, $63 \%$ received an MRI, 32\% a CT scan, and $16 \%$ had both. Idiopathic generalized epilepsies and other forms of epilepsy were evaluated with imaging studies in $50 \%$ and $87 \%$ of cases, respectively. Neuroimaging was positive for etiologically relevant abnormalities in $12.7 \%$ of cases. Of 14 patients whose clinical findings were otherwise completely normal, neuroimaging showed tuberous sclerosis in 4, tumors (2), arteriovenous abnormality (1), cavernous angioma (1), cerebral malformations (3), and other lesions (3). Seizures were partial in $13(93 \%)$ of the 14 cases, and EEGs were focal in 12 (96\%). (Berg AT, Testa FM, Levy SR, Shinnar S. Neuroimaging in children with newly diagnosed epilepsy: a community-based study. Pediatrics September 2000;106:527-532). (Reprints: Anne T. Berg PhD, Department of Biological Sciences, Northern Illinois University, DeKalb, IL 60115).

COMMENT. Etiologically relevant cerebral abnormalities may be detected by imaging studies in a small but significant proportion of newly diagnosed epilepsies in children, despite an otherwise normal clinical presentation. Patients with neurologic deficits, partial seizures, or focal EEG abnormalities are especially at risk for positive imaging studies.

\section{ELECTRICAL STATUS EPILEPTICUS DURING SLEEP}

Eighteen children with medically refractory seizures, language deterioration, incoordination, and regression in behavior and intellect, and an EEG showing electrical status epilepticus during sleep (ESES), are reported from Beijing Medical University, and the University of Hong Kong, Republic of China. Age at seizure onset ranged from 7 months to 9 years, and ESES was diagnosed at $8.5+/-4.3$ years (range $=3-21$ yrs) after onset of seizures. Development of ESES signaled the appearance of neuropsychological, language, and behavioral deterioration. Underlying epileptic syndromes included Lennox-Gastaut (4), Landau-Kleffner (3), benign rolandic epilepsy variant (5), and epileptiform autistic regression in 3,1 having Rett syndrome. Treatment with oral or intravenous clonazepam resulted in improvement in clinical and EEG manifestations, especially when treated within 2 years of seizure onset. Valproic acid was also effective in one half the patients, while carbamazepine, phenytoin, and phenobarbital were without benefit. Improvements in speech and cognitive function were correlated with suppression of ESES. (Liu XY, Wong V. Spectrum of epileptic syndromes with electrical status epilepticus during sleep in children. Pediatr Neurol May 2000;22:371-379). (Respond: Professor Virginia Wong MD, Division of Neurodevelopmental Paediatrics, Queen Mary Hospital, University of Hong Kong, Hong Kong, Peoples Republic of China).

COMMENT. The EEG phenomenon of electrical status epilepticus during sleep (ESES) in childhood may be associated with epileptic syndromes of various etiologies and outcomes. Benign rolandic epilepsy and Lennox-Gastaut syndromes represent the benign and malignant ends of the spectrum. The authors emphasize the importance of sleep EEG monitoring in children with an early onset of epilepsy associated with language, behavioral or psychological deterioration. Early treatment with oral benzodiazepines, especially clonazepam or clobazam, 